See discussions, stats, and author profiles for this publication at: https://www.researchgate.net/publication/327852259

\title{
Accurate methodology to determine slip velocity, yield stress and the constitutive relation for molten chocolate
}

Article in Journal of Food Engineering · September 2018

DOI: 10.1016/j.jfoodeng.2018.09.031

CITATIONS

3

7 authors, including:

Emeline Talansier

Université Grenoble Alpes

14 PUBLICATIONS 389 CITATIONS

SEE PROFILE

Roux D

Université Grenoble Alpes - UGA

41 PUBLICATIONS 1,282 CITATIONS

SEE PROFILE

Some of the authors of this publication are also working on these related projects:

Project Injection molding View project

Project Clotting, fibrin formation View project
READS

235

Francois Caton

Laboratoire Rhéologie et Procédés

38 PUBLICATIONS 945 CITATIONS

SEE PROFILE 


\title{
Accurate methodology to determine slip velocity, yield stress and the constitutive relation for molten chocolate
}

\author{
Emeline Talansier ${ }^{\mathrm{a}, ~ *}$, Audrey Bacconnier ${ }^{\mathrm{a}}$, François Caton ${ }^{\mathrm{a}}$, Carine Chastel ${ }^{\mathrm{a}}$, Lucy Costa ${ }^{\mathrm{a}}$, Deniz Z. Gunes ${ }^{\mathrm{b}}$, \\ Denis C.D. Roux ${ }^{\mathrm{a}, *}$ \\ ${ }^{a}$ Univ. Grenoble Alpes, CNRS, Institute of Engineering Univ. Grenoble Alpes Grenoble INP, LRP, F-38000, Grenoble, France \\ ${ }^{\mathrm{b}}$ Nestlé Research Center, Institute of Material Science, Vers-chez-les-Blanc, CH-1000, Lausanne 26, Switzerland
}

\section{A R T I C L E IN F O}

\section{Keywords:}

Yield stress fluid

Slip velocity

Solid-liquid transition

Herschel-bulkley model

Casson model

Molten chocolate

\begin{abstract}
A B S T R A C T
Concentrated soft materials like molten chocolate often has the ambiguity of behaving like a solid or a liquid depending on the external constraints. Determination of the constitutive relation is often unreliable and consequently the yield stress value too. This difficulty of determining yield stress, particularly when using a standards protocol such as the IOCCC for chocolate, is a consequence of the presence of wall slip. Assessment of slip velocity using Yoshimura and Prud'homme's method most often leads to erroneous results. We propose to tackle this problem by using an easy and reliable methodology applied to a commercial chocolate in a melted state with, as equipment, a plate-plate rotational rheometer.

This method allows precise determination of the yield stress value, the constitutive relation and the slip velocity law. Furthermore, it provides an explanation to the apparent Newtonian plateau and thickening behavior of the raw flow curve.
\end{abstract}

\section{Introduction}

Molten chocolate, a concentrated suspension of large and solid particles within a continuous fat system, is known to have a non-Newtonian behavior. Sedimentation, wall slip or solid to liquid transition occur in such complex systems (Barnes, 1995; El Kissi et al., 2006) making their rheological characterization difficult although this is essential for the sizing of industrial processes.

However, proper flow characterization is of critical importance for industrial process design, as well as for determining the best adapted formulation window for a given factory process. In the food industry, this is particularly true for chocolate manufacture, since most industrial processes have been optimized since a long time ago. In this context, the International Office of Cocoa, Chocolate and Confectionary office (IOCCC) and the International Chocolate Awards (ICA) organizations have developed specific protocols for molten chocolate (Afoakwa et al., 2009; Beckett, 2000). This protocol, currently accepted as an accepted standard, consists of successive increasing and decreasing shear rate ramps set at a series of values between 2 and $50 \mathrm{~s}^{-1}$ (see Table 1a).
Using either a Casson or an Herschel-Bulkley model, the fit of the experimental data gives the parameters of a constitutive equation (Steffe, 1996). Among these parameters, the yield stress $\sigma_{0}$ below which the material behaves as a solid, is an important parameter difficult to determine. From the IOCCC standard method the following question arises: "Why are only shear rates higher than $2 \mathrm{~s}^{-1}$ used to characterize liquid chocolate?". This is quite surprising since it is well-known that lower shear rates, below $2 \mathrm{~s}^{-1}$, are today easily attainable with any commercial rheometer.

The answer is certainly hidden within the complex solid to liquid transition of concentrated particles systems for which a resurgence of interest has been seen in the scientific literature (Balmforth et al., 2014; Bonn et al., 2015; Bonn and Denn, 2009; Damianou et al., 2014; Divoux et al., 2011; Grenard et al., 2014; Herzhaft, 2002; Herzhaft et al., 2005; Piau, 2007; Salmon et al., 2003; "Special Issue on Yield Stress Fluids: a 100 Years after Bingham's Landmark Paper," 2017). However, even if our understanding of the solid to liquid transition is gradually improving, few works have been reported on industrial soft condensed matter. Moreover, all the works that have shown interest in

\footnotetext{
* Corresponding authors. (D.C.D. Roux)

Email addresses: emeline.talansier@univ-grenoble-alpes.fr, denis.roux@univ-grenoble-alpes.fr (E. Talansier); emeline.talansier@univ-grenoble-alpes.fr, denis.roux@univ-grenoble-alpes.fr 
Table 1

(a) IOCCC2000 and ICA standards. (b) Rheometrical protocol and corresponding time sequences.

\begin{tabular}{|c|c|c|c|c|}
\hline & $\begin{array}{l}\text { Pre- } \\
\text { shear }\end{array}$ & $\begin{array}{l}\text { Increasing } \\
\text { shear rate }\end{array}$ & $\begin{array}{l}\text { Constant high } \\
\text { shear rate }\end{array}$ & $\begin{array}{l}\text { Decreasing } \\
\text { shear rate }\end{array}$ \\
\hline $\begin{array}{r}\text { IOCCC } \\
2000\end{array}$ & $\begin{array}{l}5 \mathrm{~s}^{-1} \text { on } \\
500 \mathrm{~s}\end{array}$ & $\begin{array}{l}2 \text { to } 50 \mathrm{~s}^{-1} \text { on } \\
180 \mathrm{~s}\end{array}$ & $50 s^{-1}$ on $60 s$ & $50-2 s^{-1}$ on $180 s$ \\
\hline ICA & $\begin{array}{l}5 s^{-1} \text { on } \\
300 s\end{array}$ & & & \\
\hline
\end{tabular}

\begin{tabular}{lll}
\hline Shear rate $\left(\mathrm{s}^{-1}\right)$ & $0.05-50$ & $50-0.005$ \\
\hline $\begin{array}{ll}\text { Number of collected data } \\
\text { Time (s) }\end{array}$ & 16 & 41 \\
& 50 s/variable & 50 s/variable from 15 to $50 \mathrm{~s}$ \\
\hline
\end{tabular}

solid to liquid transition face the issue of material slip at the solid wall boundaries.

Wall slip velocities are usually quantified by means of capillary rheometry (Mooney, 1931) applied to Rabinowitsch-corrected data. Using the same hypothesis as Mooney, Yoshimura and Prud'homme (Yoshimura and Prud'homme, 1988) developed a fast method based on two sets of experiments to assess wall slip using rotational rheometry. Yilmazer and Kalyon (1989) generalized this method by applying the Mooney plots procedure to parallel disk geometry and obtained a better value for wall slip velocity. Nonetheless, the Yilmazer generalization on rotational rheometry has never been assessed on yield stress fluids with the exception of Bertola's work (Bertola et al., 2003) on aqueous foam and emulsion.

Nevertheless, we should also notice that in liquid chocolate, yield stress determination using the Vane or Couette geometries along with the IOCCC standard method does not take into account the slippage of the material at the wall boundary (Aeschlimann and Beckett, 2000; Afoakwa et al., 2009, 2008; Servais et al., 2003; Sokmen and Gunes, 2006).

In the present paper we present and discuss the applicability of conventional rotational rheometry methods for accurate quantification of wall slip velocity and yield stress determination in a commercial molten chocolate. Firstly, we review protocols and cautions for accurate and reproducible rheological measurements to characterize viscoplastic molten chocolate, with an imposed torque rheometer. Then, we present the shear stress and shear rate corrections of raw data in order to obtained viscosity and slip velocity profiles, which are compared to those obtained with the Yoshimura and Prud'homme's method.

\section{Materials and methods}

\subsection{Composition and samples preparation}

"Couverture" chocolate containing 64.5\% (w/w) cocoa, 27\% sugar and $8 \%$ fat from the company Barry Callebaut was used. Emulsifier soya lecithin and natural vanilla flavor completed the $0.5 \%$ of the remaining components of the mix. This highly fluid chocolate is usually used for molded, coating or tablet use.

Molten chocolate samples were put into a sealed glass bottle placed onto a rotating mixer (RM5) inside a Memmert thermostatic chamber (Model UFE $600,256 \mathrm{~L})$. The chamber was maintained at $\mathrm{T}=50^{\circ} \mathrm{C}($ $\pm 0.5^{\circ} \mathrm{C}$ ) for a minimum period of two days. This protocol ensured that the chocolate was fully liquid and free of any remaining crystals. This preparation acted as the thermal and sample homogenization step.

\subsection{Rheometry}

Rheological measurements were performed using an imposed torque rheometer MCR501 (Anton Paar, GmbH) with a minimum torque of $0.1 \mu \mathrm{Nm}$ and a minimum rotational velocity of $10^{-6} \mathrm{rpm}$. A stainless steel, smooth plate-plate geometry of $25 \mathrm{~mm}$ diameter was employed on the rheometer with different gaps of $0.25,0.5,0.75,1.00$, $1.25,1.50,1.60,1.70$ and $1.80 \mathrm{~mm}$. In accordance with the continuum mechanics hypothesis, all gaps were much larger than the largest particles in suspension $\left(d_{\max } \approx 20 \mu \mathrm{m}\right)$ in this kind of molten chocolate (Beckett, 2000). All measurements were performed at $40^{\circ} \mathrm{C}$, using the rheometer's Peltier system. To avoid temporal behavior on creep flow at low stresses, but also aging or complex dynamics induced by residual stresses, thixotropy and retarded viscoelasticity (Benmouffok-Benbelkacem et al., 2010; Caton and Baravian, 2008; Lidon et al., 2017), measurements were conducted by imposing successive increasing and decreasing shear rate ramps as detailed in Table $1 \mathrm{~b}$ and shown in Fig. 1. Increasing shear rate ramps are known to be different from decreasing ones, as illustrated in Fig. 1 by the two first ramps of 0 and $5 \mathrm{~min}$. Indeed, when passing from solid to liquid state, yield stress fluids may have several different behaviors (Cheddadi et al., 2012; Saramito, 2007) that make the exploitation of increasing ramps impossible. Increasing ramps were only used to fluidize material and the point settings were therefore fewer than those of the decreasing ramps. If compared with the standards presented in Table 1a, the proposed decreasing ramps attained lower shear rates, as low as $0.005 \mathrm{~s}^{-1}$, thus permitting an accurate determination of the constitutive relation parameters.

Each measured point was accepted as valid if the comparison of five consecutive recordings were within a tolerance limit of $2 \%$ within a time interval of $50 \mathrm{~s}$ maximum (cf Table $1 \mathrm{~b}$ ). If the tolerance limit was over $2 \%$, the last value measured (at $50 \mathrm{~s}$ ) was selected. This protocol ensured to obtain steady state curve. In addition, measurements were made at minimum in triplicate giving a maximum experimental error of $11 \%$. The middle ramp was only selected for further exploitation in the case of perfect replicate superimposition implying that the surrounding ramps have been used as references.

By assuming either sugar and cocoa particles to be spheres of diameter $d_{\text {par } t} \approx 20 \mu \mathrm{m}$ with a density of $\rho_{\text {part }} \sim 1500 \mathrm{~kg} / \mathrm{m}^{3}$ dispersed in a continuous fat cocoa butter phase at $40^{\circ} \mathrm{C}\left(\rho_{\text {cont }}=900 \mathrm{~kg} / \mathrm{m}^{3}, \mu_{\text {cont }}\right.$ $=100$ Pas), a quick estimate of the sedimentation velocity, assuming no inter-particle interactions, $v_{\text {sed }}$ is given in the order of $5.10^{-8} \mathrm{~m} / \mathrm{s}$

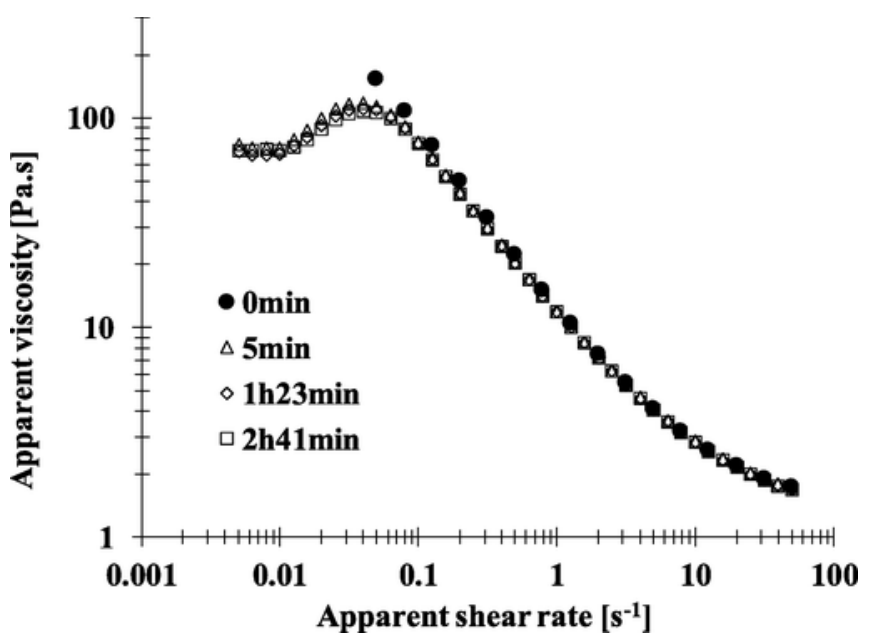

Fig. 1. Apparent viscosity $v s$ apparent shear rate evolutions at different times for the same molten chocolate sample measured with a $1 \mathrm{~mm}$ gap smooth plate-plate. Solid dots ( $0 \mathrm{~min})$ represent the first increasing ramp. Open symbols: triangles ( $5 \mathrm{~min})$, diamonds ( $1 \mathrm{~h} 23 \mathrm{~min})$ and squares $(2 \mathrm{~h} 41)$, represent decreasing ramps. The symbols show respectively steps number $1,2,6$ and 10 of the proposed protocol. 
by:

$v_{\text {sed }}=\frac{d_{\text {part }}\left(\rho_{\text {part }}-\rho_{\text {cont }}\right) g}{18 \mu_{\text {cont }}}$

The corresponding sedimentation time $h / v_{\text {sed }}$ inside the plate-plate geometry is therefore around $50 \mathrm{~h}$ for a $1 \mathrm{~mm}$ gap width. Knowing that an increasing-decreasing shear rates ramps cycle takes about $38 \mathrm{~min}$, we can deduce that no sedimentation took place in our experiments. This is confirmed by the perfect superimposition of all the curves in Fig. 1.

Finally, the normal force, i.e. perpendicular to the rotation plane, will not be displayed here as the entire measurement campaign values remained within the $15 \pm 10 \mathrm{mN}$ interval.

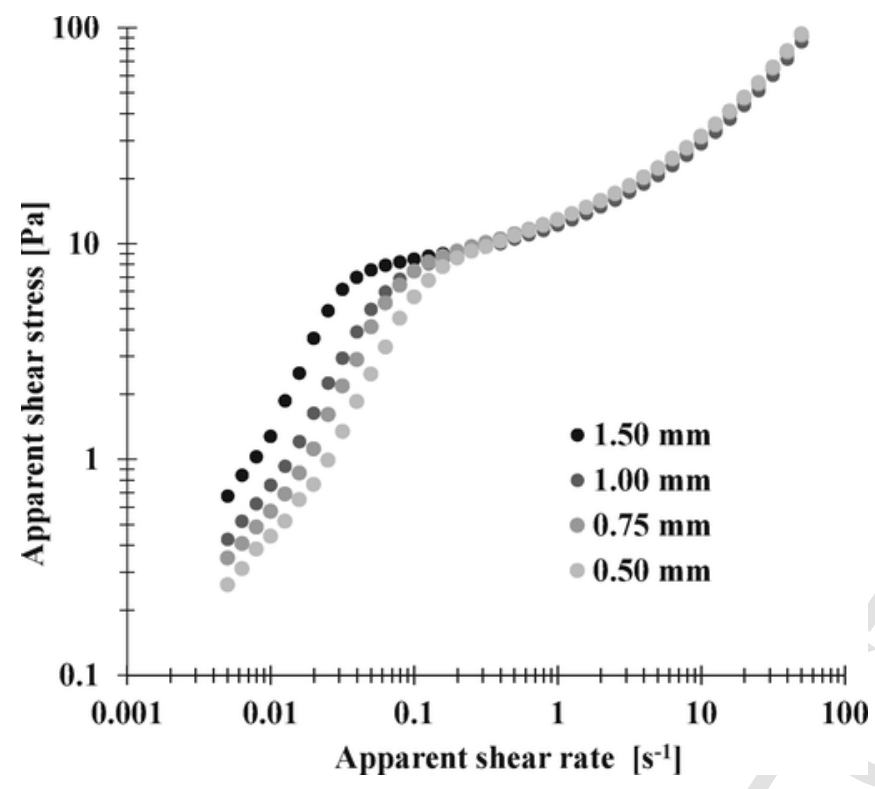

Fig. 2. Examples of apparent shear stress $v s$ shear rates curves obtained at $1.50 \mathrm{~mm}$, $1.00 \mathrm{~mm}, 0.75 \mathrm{~mm}$ and $0.50 \mathrm{~mm}$ gaps.

\section{Results and discussion}

$1 \mathrm{~mm}$-gap raw flow curves of Fig. 1 showed an apparent Newtonian plateau followed by an apparent shear-thickening and a shear-thinning behavior when increasing shear rate values. If the shear stress $v s$ shear rates are represented (Fig. 2), one can see distinct but slightly curved lines in the lower part of the graph that change abruptly into a further much more accentuated curve. The pseudo Newtonian plateau and shear-thickening behavior in Fig. 1 correspond to the lower part of the graph in Fig. 2 while the shear-thinning behavior in Fig. 1 is illustrated by the more accentuated curve in the second figure. At other gap values, similar behaviors were observed albeit with an abrupt change observed for the lower shear rate when increasing the gap size.

This shear stress $v s$ apparent shear rates representation has strong similarities to other concentrated suspensions or complex yield stress fluids, for instance, gels (Clasen, 2012; Meeker et al., 2004a, 2004b), mayonnaise (Clasen et al., 2006), fiber pulp suspensions (Derakhshandeh et al., 2010), toothpaste (Ardakani et al., 2011), shaving foam, emulsions (Bertola et al., 2003) or liquid foams (Laporte et al., 2015). This type of behavior, for which the measurements are well superimposed at high values, while remaining highly dependent on the characteristic gap length at low values is usually interpreted as the presence of a fluid wall slip (Bertola et al., 2003; Cloitre and Bonnecaze, 2017; El Kissi et al., 2006; Hatzikiriakos, 2015; Meeker et al., 2004a, 2004b; Poumaere et al., 2014; Sochi, 2011).

\subsection{Corrections of rheometrical raw data}

\subsubsection{Shear stress correction}

The science of rheology is based on the experimental determination of the material constitutive equation that links shear rate and shear stress, respectively imposed and measured by the rheometer. In the simplest shear flow configuration, the fluid is contained and sheared between two parallel circular plates in relative rotation, at a velocity $\Omega$ [rad.s $\mathrm{s}^{-1}$ ] around the vertical axis $(\mathrm{Oz})$ and separated by a distance $\mathrm{H}$ (Fig. 3a).

In plate-plate geometry, a homogeneous fluid shows a velocity field reduced to its tangential component $v_{\theta}(r, z)$, which depends solely on (a) (b)

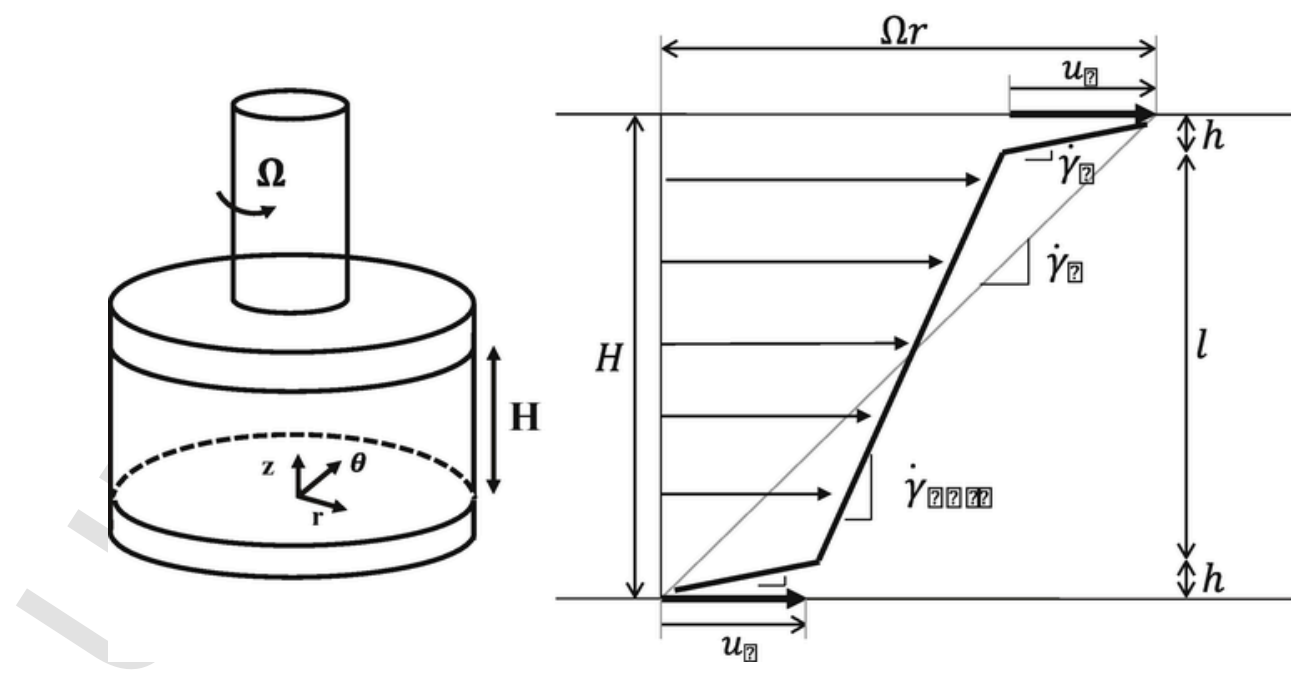

Fig. 3. (a) Schematic drawing of parallel plate-plate geometry. (b) Focus on the velocity field inside the geometry gap in the presence of slip at the solid wall. 
the distance to the rotational axis, $r$, and on the height in the gap, $\mathrm{z}$ :

$v_{\theta}(r, z)=\Omega r z / H$

The corresponding apparent local shear rate is expressed as:

$\dot{\gamma}_{a}(r)=\frac{d v_{\theta}}{d z}=\frac{\Omega r}{H}$

Inside the gap, the shear stress $\tau(r)$ is related to the torque $\Gamma$ as follows:

$\Gamma=\int_{0}^{R} 2 \pi r^{2} \tau(r) d r$

Considering Eq. (3), the torque may be rewritten as:

$\Gamma=\frac{2 \pi H^{3}}{\Omega^{3}} \int_{0}^{\dot{\gamma}_{a}(R)} \dot{\gamma}_{a}^{2} \tau\left(\dot{\gamma}_{a}\right) d \dot{\gamma}_{a}$

and at the rim of plate-plate geometry, the differentiation of Eq. (5) finally gives (Mooney, 1931; Rabinowitsch, 1929; Yilmazer and Kalyon, 1989):

$\tau_{R}=\tau\left(\dot{\gamma}_{a}(R)\right)=\left(3+\frac{d \ln \Gamma}{d \ln \dot{\gamma}_{a}(R)}\right) \frac{\Gamma}{2 \pi R^{3}}$

In all these equations, the " $a$ " and " $R$ " indices are representative of the words "apparent" and "rim". In the case of Newtonian viscous fluid, the raw shear stress, which may also be called apparent shear stress, is expressed as:

$\tau_{\text {app }}\left(\dot{\gamma}_{a, R}\right)=\tau_{\text {Newtonian }}\left(\dot{\gamma}_{a, R}\right)=\frac{2 \Gamma}{\pi R^{3}}$

At this stage, we can report that a similar correction on a Non-Newtonian aqueous hydroxyethyl cellulose solution directly carried on the viscosity was published by Krieger in 1966 (Krieger, 1966). This alternative yields corrected viscosity values obviously identical to those obtained via Eq. (6).

In Fig. 4, two sets of selected data are represented symbolized by solid dots and solid lines. The solid dots represent the mean values of the triplicates of the raw (or apparent) data previously introduced in Fig. 2.

The solid lines are the result of the shear stress correction calculated by using Eq. (6). Cubic smoothing spline "csaps" interpolations (using Matlab software) were also applied in order to compute the triplicate mean values and allow further constant shear stress calculations. At high shear stresses (i.e. high shear rates) the raw data is seen above the corresponding solid lines, reflecting an overestimation without correction. Also, a more complete overlapping at high values after correction can be seen. Conversely, at low shear stresses, the raw data is below the corresponding solid lines leading to an underestimation of the apparent shear stress without correction.

\subsubsection{Shear rate correction}

Once the shear stress had been corrected, attention was focused on the correction of the shear rate values. To do this, the sample inside the plate-plate tool illustrated in Fig. 3 was used again as follows. With a motionless lower plate, the upper plate was rotated around the $(\mathrm{Oz})$ axis at a rotation speed $\Omega$. Where there was presence of slippage phenomena at the solid walls, i.e. at $\mathrm{z}=0$ and $\mathrm{z}=\mathrm{H}$ in Fig. $3 \mathrm{~b}$, the appar-

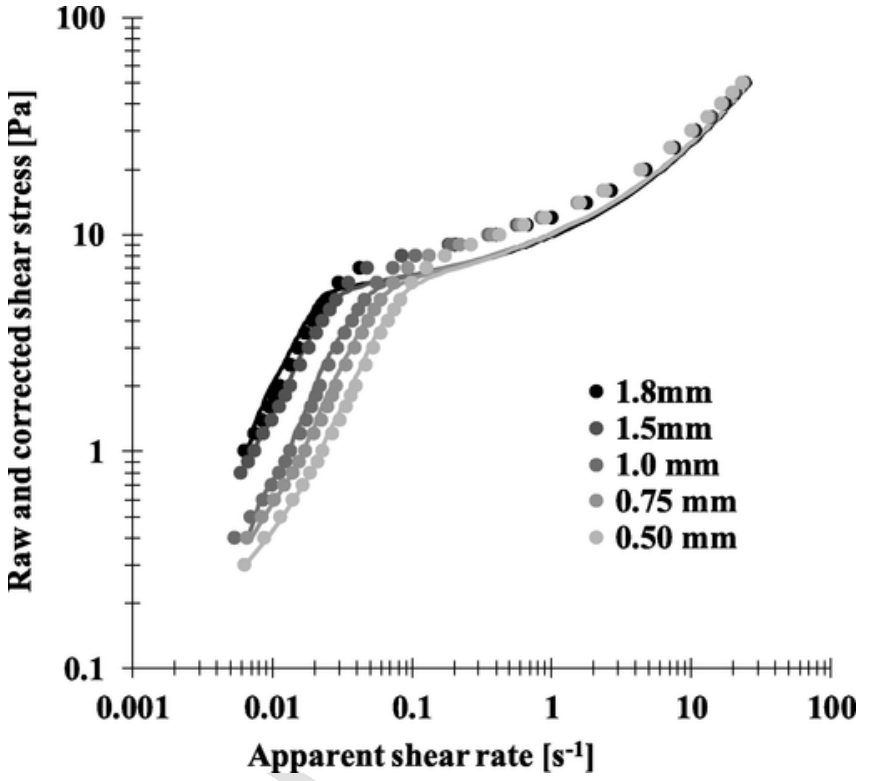

Fig. 4. Raw (solid dots) and corrected (solid lines) shear stress versus apparent shear rate curves. The solid dots correspond to mean values of interpolated raw data and solid lines to the mean values of both corrected and interpolated data. All experiments were carried out with a smooth plate-plate geometry at varying gap size: $1.80 \mathrm{~mm}, 1.50 \mathrm{~mm}, 1.00 \mathrm{~mm}$, $0.75 \mathrm{~mm}$ and $0.50 \mathrm{~mm}$.

ent shear rate was no longer homogeneous. This wall slippage can be understood macroscopically as the effect of a thin depleted layer at the solid walls sandwiching the bulk material (Barnes, 1995; Cloitre and Bonnecaze, 2017; Meeker et al., 2004a, 2004a). This depleted layer of thickness, $h$, is submitted to a high shear rate near the solid walls whereas on the bulk length, $l$, the rest of the fluid remains less sheared.

Based on Mooney's pioneer work (Mooney, 1931), the apparent slip velocity $u_{s}(r)$ at the solid walls is expressed as:

$\Omega r=\dot{\gamma}_{\text {bulk }}(r) l+2 \dot{\gamma}_{s}(r) h=\dot{\gamma}_{\text {bulk }}(r) l+2 u_{s}(r)$

where $\dot{\gamma}_{\text {bulk }}$ and $\dot{\gamma}_{s}$ are the shear rates in the bulk and in the depleted layer, respectively. A schematic drawing of the slip between the plates can be seen in Fig. 3b.

Assuming $h \ll l$ and $h \ll H$, with $H=2 l+h$ is the total length between the two plates, the apparent shear rate at the $\operatorname{rim} \dot{\gamma}_{a}(R)$, can be written as:

$\dot{\gamma}_{a}(R)=\frac{\Omega R}{H}=\dot{\gamma}_{b u l k}(R)+\frac{2 u_{s}(R)}{H}$

Using the fact that the constitutive equation links the shear stress and the shear rate, Eq. (9) can also be written as:

$\dot{\gamma}_{a}\left(\tau_{R}\right)=\frac{\Omega R}{H}=\dot{\gamma}_{b u l k}\left(\tau_{R}\right)+\frac{2 u_{s}\left(\tau_{R}\right)}{H}$

where $\dot{\gamma}_{b u l k}\left(\tau_{R}\right)$ is the bulk shear rate at a given wall stress value, $\tau_{R}$, and $u_{s}\left(\tau_{R}\right)$ is the wall slip velocity at the same shear stress. In Eq. (10), the bulk shear rate and the slip velocity are expressed at a fixed shear stress, $\tau_{R}$. In the rheological trials conducted here, all measurements were handled at fixed shear rates. As previously mentioned (§ 3.1.1), all shear stress data was corrected and interpolated with a "csaps" tool in order to use Eq. (10) at $\tau_{R}$. 
Fig. 5a-c details the apparent shear rate $v s$ the reciprocal gap at different shear stresses in order to compare experimental data in the model expressed by Eq. (10). Fig. 6 gives a summary of the different behaviors detailed below.

This representation of the apparent shear rate $v s 1 / \mathrm{H}$ has been used for highly filled suspensions in the past (Yilmazer and Kalyon, 1989). As illustrated by Fig. $5 \mathrm{a}$ and $\mathrm{b}$ and $5 \mathrm{c}$, our data has been split into three different plots.

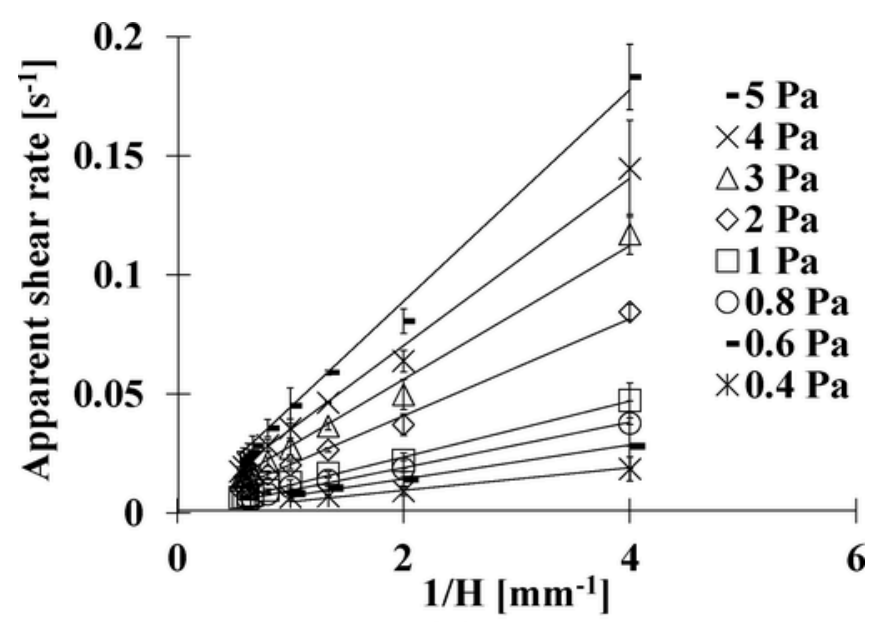

(a)

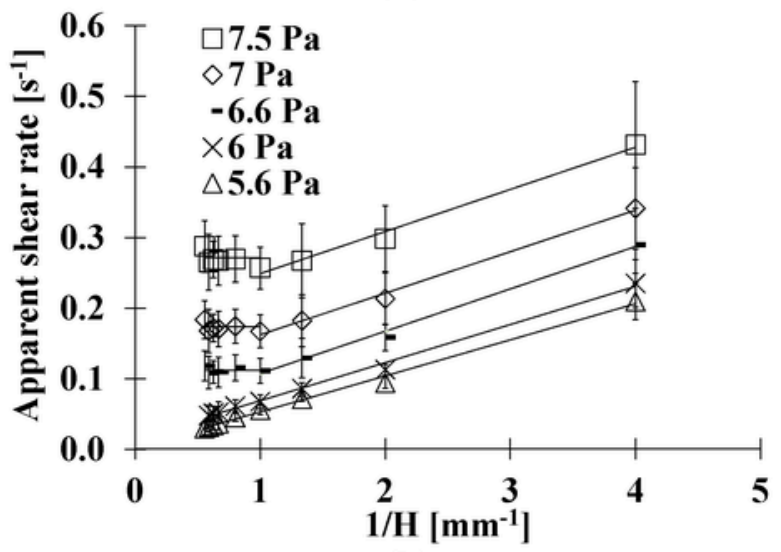

(b)

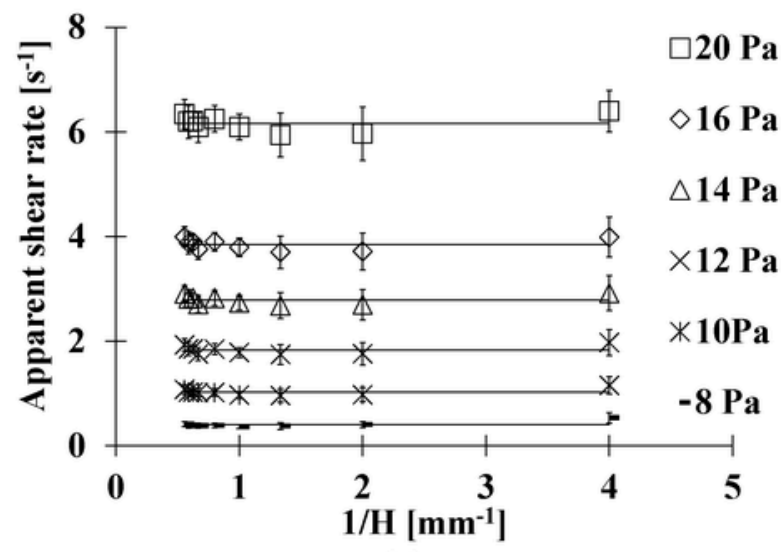

(c)

Fig. 5. Apparent shear rate as a function of the reciprocal gap for different constant shear stresses. Details of each encountered behavior: (a) low shear rate: pure slip regime, (b) intermediate shear rate: transition regime (c) high shear rate: homogeneous bulk shear regime.
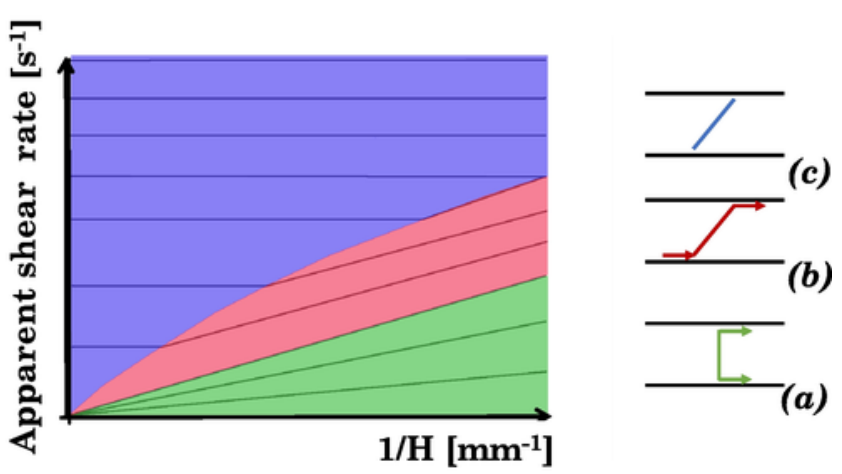

(a)

Fig. 6. Apparent shear rate as a function of the reciprocal gap for different constant shear stresses represented by the solid lines. A summary of the different behaviors encountered on the left is symbolized by the three representations on the right: (a) at low shear rate (in green), a pure slip regime, (b) at intermediate shear rate (in red), a transition regime (c) at high shear rate (in blue), a homogeneous bulk shear regime.

In Fig. 5a, for low shear stresses, the apparent shear rates are shown to be strictly proportional to $1 / \mathrm{H}$. According to Eq. (10), the best fit of experimental data is reduced to an affine function where only wall slip occurs (Fig. 6, green region). The apparent shear of the sample is then reduced to a solid-like slippage at the wall, whereas the "fluid" bulk remains unsheared, but only elastically distorted.

In Fig. $5 c$, for high shear stresses, apparent shear rates are represented by the horizontal straight lines (Fig. 6, blue region). Thus, in Eq. (10) the bulk shear rate $\dot{\gamma}_{\text {bulk }}\left(\tau_{R}\right)$ contribution largely exceeds the terms of slippage $\frac{2 u_{S}\left(\tau_{R}\right)}{H}$ implying equality between applied and measured shear rates.

In Fig. 5b, for intermediate shear stresses, the apparent shear rates are no longer proportional to $1 / \mathrm{H}$ but, rather, composed of two straight lines joined at $1 / \mathrm{H}=1.33 \mathrm{~mm}^{-1}$ (or $0.75 \mathrm{~mm}$ equivalent gap), therefore implying two ranges for the use of Eq. (10). At 1/H values below $1.33 \mathrm{~mm}^{-1}$ (blue region, Fig. 6) the chocolate behaves as a liquid-like material without any slip velocity. This behavior is identical to that of Fig. $5 c$, where the slip velocity term in Eq. (10) is negligible. Above $1.33 \mathrm{~mm}^{-1}$ (red region), the chocolate may take on a partially liquid and also a partially solid form so both the two terms of Eq. (10) must be considered when data are adjusted. For instance, at $6.6 \mathrm{~Pa}$, the fit of experimental data gives the slip velocity $u_{s}$ equal to $0.03 \mathrm{~m} / \mathrm{s}$ and a bulk shear rate $\dot{\gamma}_{\text {bulk }}$ of $0.05 \mathrm{~s}^{-1}$. The reciprocal length $1 / \mathrm{H}=1.33 \mathrm{~mm}^{-1}$ is consequently the separating line between these two different behaviors or regimes and is surely a consequence of confinement of the particles, which measure approximately $20 \mu \mathrm{m}$. This same transition $1 /$ $\mathrm{H}=1.33 \mathrm{~mm}^{-1}$ corresponds to $\mathrm{H}=750 \mu \mathrm{m}$, indicating that the gap is only $\sim 40$ times the size of an average particle and therefore relatively small. This value seems to be the limit for distinguishing between an "equivalent granular" or alternatively a continuous view of the molten chocolate used here. The "equivalent granular" material flows more like a solid while the continuous material will flows more like a liquid (Liu and Nagel, 1998). Without going further in the interpretation of such behavior, it can be argued that the transition driven by the $1 / \mathrm{H}$ value, i.e. by the gap size, is due to a localized "cooperative flow event". Such an event affects the fluidity, which is highly dependent on the ratio of $\mathrm{H}$ to particle size (Coussot, 2014; Goyon et al., 2010).

A second important parameter that drives chocolate flow or not is the experiment duration. For experiments of short duration, the material will be elastically distorted but will not flow. For longer experiments, the material will first be distorted and then, depending on the stress applied, will flow after either a short or a substantially longer time as shown in the existing literature (Auffret et al., 2009; Benmouffok-Benbelkacem et al., 2010; Caton and Baravian, 2008; 
Cheddadi et al., 2012; Coussot, 2014; Ovarlez, 2011; Ovarlez and Chateau, 2008; Saramito, 2007).

To conclude this section, Fig. 6 summarizes what must be borne in mind and what interpretation is to be given to the symbols on the right. For low shear stresses (in green), the chocolate behaves as a solid that slips at the wall (insert (a), Fig. 6). For high or intermediate shear stresses and small $1 / \mathrm{H}$ (in blue), the chocolate behaves as a liquid-like material without any slip velocity at the walls (insert (c), Fig. 6). For intermediate shear stresses (in red), the chocolate behaves partially as liquid and also partially as solid material showing simultaneously the behaviors of both bulk flow and slip at the walls (insert (b), Fig. 6). This latter case is interpreted to be a consequence of the non-homogeneous shear rate distribution within the gap induced by the progressive fluidization of the yield stress fluid.

As a consequence, a compromise has to be found between the required level of characterization and the corresponding period available to carry out the assays. In fact, the cost necessary to accurately determine the yield stress value will be proportional to the measurement duration. From an industrial point of view, applying the present method to a few well selected gap values, e.g. two or three, may be sufficient, particularly if a well-defined programming procedure is used. By contrast, to distinguish between products possessing very similar behaviors, e.g. two molten chocolates of similar composition and particle size, a longer duration and a greater number of experiments will be required.

From a scientific point of view, the generalization of this method could help to provide some clues to a deeper understanding of flow types close to the yield stress. In addition, the tool presented here can also be used in numerical studies to set the boundary conditions at solid walls.

\subsubsection{Conventional Yoshimura and Prudhomme correction}

Based on the continuum hypothesis of shear stress within the gap (cf 3.1.1), the conventional Yoshimura and Prudhomme method (Yoshimura and Prud'homme, 1988) proposed the use of two series of experimental data carried out at $\mathrm{H}_{1}$ and $\mathrm{H}_{2}$ gap sizes, which allows the calculation of the apparent shear rate and viscosity at a given shear stress:

$\dot{\gamma}_{a R}\left(\tau_{R}\right)=\frac{H_{1} \dot{\gamma}_{a R 1}\left(\tau_{R}\right)-H_{2} \dot{\gamma}_{a R 2}\left(\tau_{R}\right)}{H_{1}-H_{2}}$

$\eta\left(\dot{\gamma}_{a R}\right)=\frac{\tau_{R}\left(H_{1}-H_{2}\right)}{H_{1} \dot{\gamma}_{a R 1}\left(\tau_{R}\right)-H_{2} \dot{\gamma}_{a R 2}\left(\tau_{R}\right)}$

Slip velocity may also be directly deduced:

$u_{s, Y \& P}\left(\tau_{R}\right)=\frac{\dot{\gamma}_{a R 1}\left(\tau_{R}\right)-\dot{\gamma}_{a R 2}\left(\tau_{R}\right)}{2\left(\frac{1}{H_{1}}-\frac{1}{H_{2}}\right)}$

Hereinafter, we will apply these formula to the interpolated and shear stress corrected data in $\S 3.1 .1$.

\subsection{Wall slip velocity}

In Fig. 7, the solid triangles represent the wall slip velocity $u_{s}(\mathrm{~mm} / \mathrm{s})$ in relation to the shear stress $\tau_{R}(\mathrm{~Pa})$. The wall slip velocity was obtained from the data represented in Fig. $5 \mathrm{a}$ and $\mathrm{c}$ and by using Eq. (10). Below 5.4Pa in the green region, the slip velocity $u_{s}$ follows an affine function of slope $u_{s} / \tau_{R}=4.55 \times 10^{-3} \pm 4.11 \times 10^{-5} \mathrm{~mm} /($

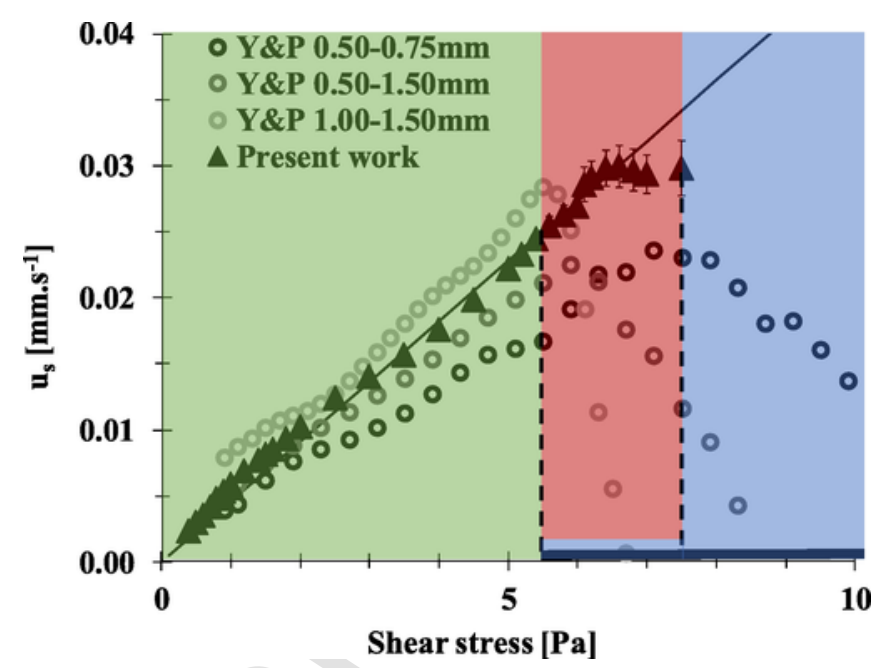

Fig. 7. Wall slip velocity as a function of the shear stress. The solid triangle symbols and the bottom thick solid line between 6 and $10 \mathrm{~Pa}$ illustrate experimental data from the present work. The solid line represents the correspondingly best linear fit $\left(u_{s}=4.55 .10^{-3} \tau_{R}\right.$ ). The open circle symbols represent the wall slip velocity evolution obtained using the Yoshimura and Prud'homme method with $0.50-0.75 ; 0.50-1.50$ and $1.00-1.50 \mathrm{~mm}$ gaps pair.

Pa.s). For these stresses, the chocolate behaves as a solid-like material. Above $8 \mathrm{~Pa}$ in the blue region, the slip velocity symbolized by the thick solid line at the bottom is negligible. In this domain, the chocolate is fully sheared without any slip velocity at the walls.

Between 5.4Pa and 8Pa, two slip velocities are obtained. For a constant shear stress at low $1 / \mathrm{H}$ values, there is no slip velocity as represented by the horizontal lines in Fig. 5b. These results are represented by the thick solid line in the blue region in Fig. 7. At high $1 / \mathrm{H}$ values, the fit of the linear shapes in Fig. 5b using Eq. (10), as previously explained, returns not only values of the bulk shear rate but also a constant slip velocity $u_{s}$ of approximately $0.03 \mathrm{~mm} / \mathrm{s}$ whatever the applied stress, as represented by the triangles in the red region in Fig. 7.

In the existing literature, slip velocity is assumed to be either proportional (Russel and Grant, 2000) or quadratically dependent on the shear stress (Clasen, 2012; Meeker et al., 2004b; Salmon et al., 2003; Yeow et al., 2006). However, at this stage there is no obvious explanation for these differences.

A path worthy of consideration could be seen in Fig. 7 by open circle symbols representing data obtained using the Yoshimura and Prud'homme method (Yoshimura and Prud'homme, 1988) applied at $0.50-0.75 \mathrm{~mm} ; 0.50-1.50$ and $1.00-1.50 \mathrm{~mm}$ gaps pair, respectively. Clearly, the Yoshimura and Prud'homme method produces different results in relation to the gaps pair used. Furthermore, the velocity curves show surprising concave shapes characterized by a maximum occurring in the intermediate shear stress region (red region). At low stresses (green region), the difficulty in reproducing the same slip velocity evaluation when the gaps pair is changed can be interpreted as the result of a combination of experimental errors and of the difference between the two gaps. At intermediate stresses (red region), the decrease in the slip velocity is induced by the use of Eq. (13) at two reciprocal gaps $1 / \mathrm{H}_{1}$ and $1 / \mathrm{H}_{2}$, within two different regions, for instance, the red region for $1 / \mathrm{H}_{1}$ and the blue region for $1 / \mathrm{H}_{2}$ in Fig. 6 . In this case, it becomes obvious that the necessity of following a constant shear stress line to join a shear rate couple, at $1 / \mathrm{H}_{1}$ and $1 / \mathrm{H}_{2}$ values respectively, will not be satisfied.

For these reasons, we do not recommend the use of the Yoshimura and Prud'homme method on all yield stress fluids in order to determine the slip $v s$ stress evolution and even less so for conditions close to the yield stress. 


\subsection{Reconstitution of the steady state bulk flow curve}

The effective viscosity $v s$ the bulk shear rate figure can now be reconstituted (Fig. 8). The effective viscosity is computed by a simple division of the corrected shear stress (\$3.1.1) by the bulk (corrected) shear rate (§3.1.2). In contrast to Fig. 1 the present work led to a yield stress fluid behavior depicted by a continuous decrease of the effective viscosity $v s$ the bulk shear rate. The apparent Newtonian plateau followed by an apparent shear-thickening behavior in Fig. 1 completely disappeared when the corrections proposed in this article were applied. Nonetheless, this is not the case for our data (open circles, Fig. 8) to which we applied the Yoshimura and Prud'homme correction. Indeed, this last figure shows a Newtonian plateau for the different selected gaps pair. Moreover, the plateau value will depend on the selected gaps pair.

Finally, Fig. 9 shows the evolution of the corrected shear stresses (§3.1.1) vs bulk shear rates (§3.1.2) evolutions in logarithmic scales. This representation is one of the most common representations of yield stress fluid.

Below a critical stress, also called bulk yield stress, of $5.4 \mathrm{~Pa}$ the shear rate is zero and no flow occurs. Up to $5.4 \mathrm{~Pa}$, the shear rate is not equal to zero and so the fluid flows, apparently with a linear stress to shear rate dependence. As the log-log scale representation expands low values, this representation is particularly suitable for focusing on the surrounding yield stress where values tend to be constant.

Additional solid lines in Fig. 9 represent the fits using conventional Casson and Herschel-Bulkley models on the shear rate range of IOCCC recommendations from 2 to $50 \mathrm{~s}^{-1}$ (broken lines) but also on the entire range of data, from 0.005 to $50 \mathrm{~s}^{-1}$ (solid lines). As a reminder, the Herschel-Bulkley (Eq. (14)) and Casson (Eq. (15)) constitutive equations are expressed as:

$\sigma=\sigma_{0, H B}+k_{H B} \dot{\gamma}^{n}$

$\sqrt{\sigma}=\sqrt{\sigma_{O, C}}+\sqrt{\eta_{C} \dot{\gamma}}$

where $\sigma_{0, H B}, k_{H B}, n$ are respectively the Herschel-Bulkley yield stress, the consistency index and the flow index parameters, on the one hand, and

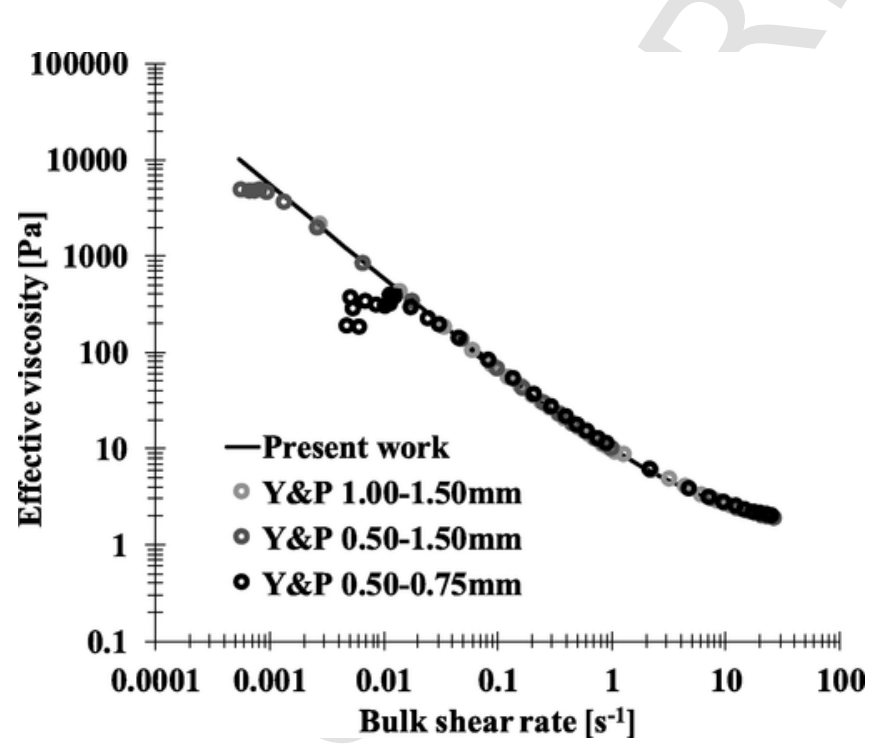

Fig. 8. Evolution of the effective viscosity $v s$ bulk shear rate. The solid line represents the present work's corrected data. Open circles represent the data obtained via the Yoshimura and Prud'homme method with 1.00-1.50; 0.50-1.50 and $0.50-0.75 \mathrm{~mm}$ gaps pair.

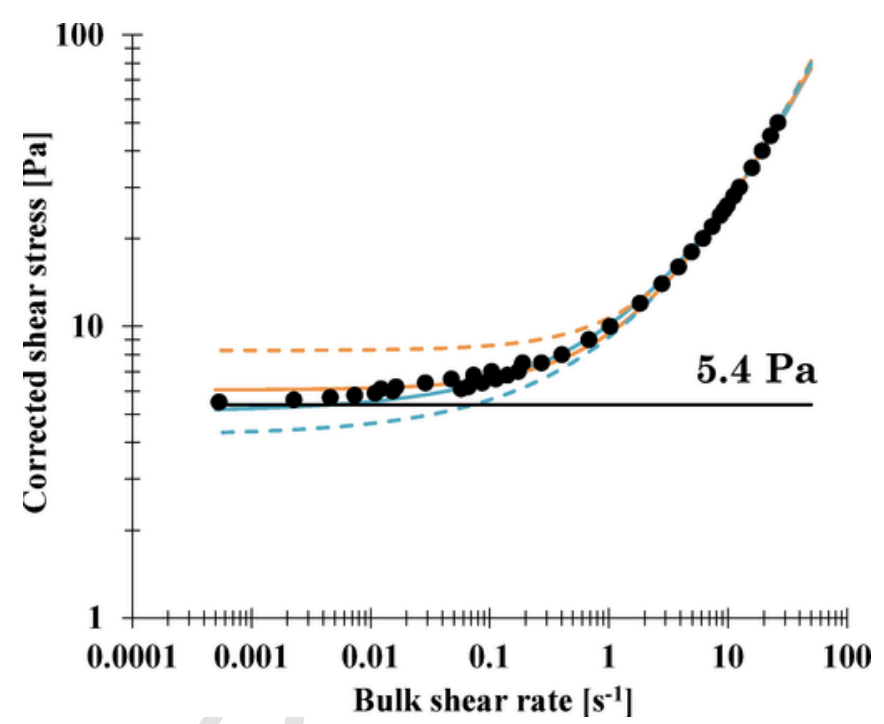

Fig. 9. Evolution of corrected shear stress $v s$ bulk shear rate (solid circle symbols). The lines correspond to the different data fittings using Casson (in blue) and Herschel-bulkley (in orange) as models. Broken lines refer to the IOCCC standard conditions, i.e. a $2 \mathrm{~s}^{-1}-50$ $\mathrm{s}^{-1}$ fitting while solid lines refer to a fit over the entire range of data, i.e. a $0.005-50 \mathrm{~s}^{-1}$ fitting.

$\sigma_{0, C}, \eta_{C}$ are respectively the Casson yield stress and the viscosity on the other hand. For information purposes, Table 2 summarizes all obtained parameters from the fitting of corrected data from the IOCCC standard conditions (2-50 s $\left.\mathrm{s}^{-1}\right)$ and also for the extended conditions (0.005-50 $\mathrm{s}^{-1}$ ) with Eq. (14) and Eq. (15).

From Table 2 and Fig. 9 and, it can be observed that the Herschel-Bulkley model is systematically above experimental data whereas the Casson model is systematically below them. Moreover, both models applied within the range of the IOCCC standard recommendation reveal a larger discrepancy. Indeed, as an example, the Herschel-Bulkley model gives a yield stress of $8.27 \pm 0.14 \mathrm{~Pa}$ rather than the $5.4 \pm 0.2 \mathrm{~Pa}$ directly determined in Fig. 5. It is slightly better for the Casson model, which gives a yield stress of $4.23 \pm 0.10 \mathrm{~Pa}$.

For both models, the viscosity, consistency and pseudo plastic index are summarized in Table 2 in addition to the yield stress value. All these estimations constitute useful data to characterize a yield stress fluid properly.

It should be noted that the evaluation of the yield stress by using both the Casson and Herschel-Bulkley models is of particular interest in the present case, as these models respectively under and overestimate

Table 2

Summary of the different parameters obtained by fitting the molten chocolate data with the Herschel-Bulkley and Casson models. The symbol * indicates deviation from the bulk yield stress 5.4 Pa determined directly in Fig.5.

\begin{tabular}{|c|c|c|}
\hline \multirow[t]{3}{*}{$\begin{array}{l}\text { Shear rate } \\
\text { range for } \\
\text { fittings }\end{array}$} & Corrected data & \\
\hline & Herschel-Bulkley & Casson \\
\hline & $\sigma=\sigma_{0, H B}+k_{H B} \dot{\gamma}^{n}$ & $\sqrt{\sigma}=\sqrt{\sigma_{C}}+\sqrt{\eta_{C} \dot{\gamma}}$ \\
\hline $\begin{array}{l}\text { Standard } \\
\text { conditions } \\
\left(2-50 \mathrm{~s}^{-1}\right)\end{array}$ & $\begin{array}{l}\sigma_{0, H B}=8.27 \pm 0.14 \mathrm{~Pa}(1.7 \%) \\
(53 \%)^{*} k_{H B}=2.38 \pm 0.06 \mathrm{~Pa} . \mathrm{s}^{\mathrm{n}} \\
(2.3 \%) n_{H B}=0.876 \pm 0.006 \\
(0.7 \%)\end{array}$ & $\begin{array}{l}\sigma_{0, c}=4.23 \pm 0.10 \mathrm{~Pa} \\
(2.3 \%)(22 \%)^{*} \\
\eta_{c}=0.94 \pm 0.01 \text { Pa s }(1.3 \%)\end{array}$ \\
\hline $\begin{array}{l}\text { Extended } \\
\text { conditions } \\
(0.005-50 \\
\left.\mathrm{s}^{-1}\right)\end{array}$ & $\begin{array}{l}\sigma_{0, H B}=6.05 \pm 0.08 \mathrm{~Pa}(1.3 \%) \\
(12 \%)^{*} k_{H B}=3.39 \pm 0.08 \mathrm{~Pa}^{\mathrm{n}}{ }^{\mathrm{n}} \\
(2.4 \%) n_{H B}=0.780 \pm 0.008 \\
(1.0 \%)\end{array}$ & $\begin{array}{l}\sigma_{0, c}=5.10 \pm 0.08 \mathrm{~Pa} \\
(1.7 \%)(5.6 \%)^{*} \\
\eta_{c}=0.85 \pm 0.01 \text { Pas }(1.3 \%)\end{array}$ \\
\hline
\end{tabular}


it. This point could be a subject worthy of further study. Finally, we can also notice that the first stress value for which the molten chocolate begins to flow in Fig. 5b (§3.1.2), represented by the horizontal line in Fig. 9 at $5.4 \mathrm{~Pa}$, is in agreement with the yield stress obtained by using the best fits.

\section{Conclusion}

In this article we have proposed a simple and reliable method particularly well-adapted for the characterization of food materials showing yield stress behavior. This method allows identification of the constitutive and slip velocity equations, and consequently an accurate evaluation of rheological parameters, essential for material characterization such as viscosity, consistency, pseudo plastic index, or yield stress. The level of accuracy of such parameters increases if, and only if, (i) an extended range of reciprocal gap is used and (ii) if all collected data are in an apparently steady state. In addition, the rheometrical steady state can be long to obtain close to the yield stress.

The originality of this method lies in its capacity to take into account the correction of the slip velocity simultaneously with the non-homogeneity of the flow field within the geometry gap. It also highlights the fact that the Yoshimura and Prud'homme method is inappropriate for yield stress fluid characterization.

Molten chocolate, given here as an example of an industrial multiphase product, was found to be a relatively simple material as it is not subject to aging, retarded viscoelasticity, thixotropy or sedimentation phenomena within the measurement duration. Nevertheless, the validity of our method remains to be demonstrated for more complex yield stress soft materials. Indeed, we believe that this method is applicable to all fluids, even complex fluids such as industrial foodstuffs, provided that they are not too heterogeneous at the scale of the geometry gap. Finally, provided that a sufficient level of accuracy is reached, one can expect to be able to distinguish molten chocolates of similar formulations or other liquid foodstuffs.

\section{Acknowledgments}

The Laboratoire Rhéologie et Procédés is part of the LabEx Tec 21 (Investissements d'Avenir - grant agreement n ${ }^{\circ}$ ANR-11-LABX-0030) and of the PolyNat Carnot Institut (Investissements d'Avenir - grant agreement $n^{\circ}$ ANR-11-CARN-030-01). Authors would like to gratefully acknowledge Susan Sinclair for proofing the manuscript.

\section{References}

Aeschlimann, J.-M., Beckett, S.T., 2000. International inter-laboratory trials to determine the factors affecting the measurement of chocolate viscosity. J. Texture Stud. 31, 541-576.

Afoakwa, E.O., Paterson, A., Fowler, M., 2008. Effects of particle size distribution and composition on rheological properties of dark chocolate. Eur. Food Res. Technol. 226, 1259-1268 https://doi.org/10.1007/s00217-007-0652-6.

Afoakwa, E.O., Paterson, A., Fowler, M., Vieira, J., 2009. Comparison of rheological models for determining dark chocolate viscosity. Int. J. Food Sci. Technol. 44, 162-167 https://doi.org/10.1111/j.1365-2621.2008.01710.x.

Ardakani, H.A., Mitsoulis, E., Hatzikiriakos, S.G., 2011. Thixotropic flow of toothpaste through extrusion dies. J. Non-Newtonian Fluid Mech. 166, 1262-1271 https://doi. org/10.1016/j.jnnfm.2011.08.004.

Auffret, Y., Roux, D.C.D., El Kissi, N., Dunstan, D.E., Pignot-Paintrand, I., 2009. Stress and strain controlled rheometry on a concentrated lyotropic lamellar phase of AOT/ Water/Iso-octane. Rheol. Acta 48, 423-432 https://doi.org/10.1007/ s00397-008-0336-6.

Balmforth, N.J., Frigaard, I.A., Ovarlez, G., 2014. Yielding to stress: recent developments in viscoplastic fluid mechanics. Annu. Rev. Fluid Mech. 46, 121-146 https://doi.org/ 10.1146/annurev-fluid-010313-141424.

Barnes, H.A., 1995. A review of the slip (wall depletion) of polymer solutions, emulsions and particle suspensions in viscometers: its cause, character, and cure. J. Non-Newtonian Fluid Mech. 56, 221-251 https://doi.org/10.1016/0377-0257(94)01282-M.

Beckett, S.T., 2000. The Science of Chocolate. Royal Society of Chemistry.
Benmouffok-Benbelkacem, G., Caton, F., Baravian, C., Skali-Lami, S., 2010. Non-linear viscoelasticity and temporal behavior of typical yield stress fluids: carbopol, Xanthan and Ketchup. Rheol. Acta 49, 305-314 https://doi.org/10.1007/s00397-010-0430-4.

Bertola, V., Bertrand, F., Tabuteau, H., Bonn, D., Coussot, P., 2003. Wall slip and yielding in pasty materials. J. Rheol. 47, 1211-1226 https://doi.org/10.1122/1.1595098.

Bonn, D., Denn, M.M., 2009. Yield stress fluids slowly yield to analysis. Science 324, 1401-1402.

Bonn, D., Paredes, J., Denn, M.M., Berthier, L., Divoux, T., Manneville, S., 2015. Yield Stress Materials in Soft Condensed Matter. ArXiv150205281 Cond-mat.

Caton, F., Baravian, C., 2008. Plastic behavior of some yield stress fluids: from creep to long-time yield. Rheol. Acta 47, 601-607 https://doi.org/10.1007/ s00397-008-0267-2.

Cheddadi, I., Saramito, P., Graner, F., 2012. Steady Couette flows of elastoviscoplastic fluids are nonunique. J. Rheol. 56, 213-239 https://doi.org/10.1122/1.3675605.

Clasen, C., 2012. Determining the true slip of a yield stress material with a sliding plate rheometer. Rheol. Acta 51, 883-890.

Clasen, C., Gearing, B.P., McKinley, G.H., 2006. The flexure-based microgap rheometer (FMR). J. Rheol. 50, 883-905 https://doi.org/10.1122/1.2357190.

Cloitre, M., Bonnecaze, R.T., 2017. A review on wall slip in high solid dispersions. Rheol. Acta 56, 283-305 https://doi.org/10.1007/s00397-017-1002-7.

Coussot, P., 2014. Yield stress fluid flows: a review of experimental data. J. Non-Newtonian Fluid Mech. 211, 31-49 https://doi.org/10.1016/j.jnnfm.2014.05.006.

Damianou, Y., Philippou, M., Kaoullas, G., Georgiou, G.C., 2014. Cessation of viscoplastic Poiseuille flow with wall slip. J. Non-Newtonian Fluid Mech. 203, 24-37.

Derakhshandeh, B., Hatzikiriakos, S.G., Bennington, C.P.J., 2010. Rheology of pulp suspensions using ultrasonic Doppler velocimetry. Rheol. Acta 49, 1127-1140 https:// doi.org/10.1007/s00397-010-0485-2.

Divoux, T., Barentin, C., Manneville, S., 2011. From stress-induced fluidization processes to Herschel-Bulkley behaviour in simple yield stress fluids. Soft Matter 7, 8409-8418.

El Kissi, N., Nigen, S., Pignon, F., 2006. Glissement et Rhéométrie. Rhéologie. 13-39.

Goyon, J., Colin, A., Bocquet, L., 2010. How does a soft glassy material flow: finite size effects, non local rheology, and flow cooperativity. Soft Matter 6, 2668-2678 https:// doi.org/10.1039/C001930E.

Grenard, V., Divoux, T., Taberlet, N., Manneville, S., 2014. Timescales in creep and yield ing of attractive gels. Soft Matter 10, 1555-1571.

Hatzikiriakos, S.G., 2015. Slip mechanisms in complex fluid flows. Soft Matter 11, 7851-7856.

Herzhaft, B., 2002. Correlation between transient shear experiments and structure evolution of aqueous foams. J. Colloid Interface Sci. 247, 412-423.

Herzhaft, B., Kakadjian, S., Moan, M., 2005. Measurement and modeling of the flow behavior of aqueous foams using a recirculating pipe rheometer. Colloids Surf. Physicochem. Eng. Asp., A collection of papers presented at the 5th European Conference on Foams, Emulsions, and Applications, EUFOAM 2004, University of Marne-la-Vallee, Champs sur Marne (France), 5-8 July, 2004, vol. 263, 153-164 https://doi.org/10. 1016/j.colsurfa.2005.01.012.

Krieger, I.M., 1966. Direct determination of the flow curves of non-Newtonian fluids. IV. Parallel-plane rotational viscometer. J. Appl. Phys. 37 (4703) https://doi.org/10. 1063/1.1708121.

Laporte, M., Della Valle, D., Loisel, C., Marze, S., Riaublanc, A., Montillet, A., 2015. Rheological properties of food foams produced by SMX static mixers. Food Hydrocolloids 43, 51-57 https://doi.org/10.1016/j.foodhyd.2014.04.035.

Lidon, P., Villa, L., Manneville, S., 2017. Power-law creep and residual stresses in a carbopol gel. Rheol. Acta 56, 307-323 https://doi.org/10.1007/s00397-016-0961-4

Liu, A.J., Nagel, S.R., 1998. Nonlinear dynamics: jamming is not just cool any more. Nature 396, 21-22.

Meeker, S.P., Bonnecaze, R.T., Cloitre, M., 2004a. Slip and flow in soft particle pastes. Phys. Rev. Lett. 92 (198302) https://doi.org/10.1103/PhysRevLett.92.198302.

Meeker, S.P., Bonnecaze, R.T., Cloitre, M., 2004b. Slip and flow in pastes of soft particles: direct observation and rheology. J. Rheol. 48, 1295-1320 https://doi.org/10.1122/1. 1795171.

Mooney, M., 1931. Explicit formulas for slip and fluidity. J. Rheol. 1929-1932 (2), 210-222 https://doi.org/10.1122/1.2116364.

Ovarlez, G., 2011. Rhéologie des pâtes granulaires (thesis). Université Paris-Est.

Ovarlez, G., Chateau, X., 2008. Influence of shear stress applied during flow stoppage and rest period on the mechanical properties of thixotropic suspensions. Phys. Rev. E 77 (061403) https://doi.org/10.1103/PhysRevE.77.061403.

Piau, J.M., 2007. Carbopol gels: elastoviscoplastic and slippery glasses made of individual swollen sponges: meso-and macroscopic properties, constitutive equations and scaling laws. J. Non-Newtonian Fluid Mech. 144, 1-29.

Poumaere, A., Moyers-González, M., Castelain, C., Burghelea, T., 2014. Unsteady laminar flows of a Carbopol ${ }^{\circledR}$ gel in the presence of wall slip. J. Non-Newtonian Fluid Mech. 205, 28-40 https://doi.org/10.1016/j.jnnfm.2014.01.003.

Rabinowitsch, B., 1929. Über die viskosität und elastizität von solen. Z. Phys. Chem. 145 , $1-26$.

Russel, W.B., Grant, M.C., 2000. Distinguishing between dynamic yielding and wall slip in a weakly flocculated colloidal dispersion. Colloid. Surf. Physicochem. Eng. Aspect. 161, 271-282.

Salmon, J.-B., Bécu, L., Manneville, S., Colin, A., 2003. Towards local rheology of emulsions under Couette flow using Dynamic Light Scattering. Eur. Phys. J. Soft Matter 10, 209-221 https://doi.org/10.1140/epje/i2002-10110-5.

Saramito, P., 2007. A new constitutive equation for elastoviscoplastic fluid flows. J. Non-Newtonian Fluid Mech. 145, 1-14 https://doi.org/10.1016/j.jnnfm.2007.04.004.

Servais, C., Ranc, H., Roberts, I. d, 2003. Determination of chocolate viscosity. J. Texture Stud. 34, 467-497 https://doi.org/10.1111/j.1745-4603.2003.tb01077.x. 
Sochi, T., 2011. Slip at fluid-solid interface. Polym. Rev. 51, 309-340 https://doi.org/10. 1080/15583724.2011.615961.

Sokmen, A., Gunes, G., 2006. Influence of some bulk sweeteners on rheological properties of chocolate. LWT - Food Sci. Technol. 39, 1053-1058 https://doi.org/10.1016/j.lwt. 2006.03.002.

Special Issue on Yield Stress Fluids: a 100 Years after Bingham's Landmark Paper, 2017. Rheological Acta vol. 56(3).

Steffe, J.F., 1996. Rheological Methods in Food Process Engineering. Freeman Press.
Yeow, Y.L., Leong, Y.-K., Khan, A., 2006. Non-Newtonian flow in parallel-disk viscometers in the presence of wall slip. J. Non-Newtonian Fluid Mech. 139, 85-92 https://doi. org/10.1016/j.jnnfm.2006.07.005.

Yilmazer, U., Kalyon, D.M., 1989. Slip effects in capillary and parallel disk torsional flows of highly filled suspensions. J. Rheol. 33, 1197-1212 https://doi.org/10.1122/ 1.550049 .

Yoshimura, A., Prud'homme, R.K., 1988. Wall slip corrections for Couette and parallel disk viscometers. J. Rheol. 32 . 